\title{
Fractura de cadera en un hospital especializado. Seguimiento a un año
}

\section{Hip fracture in a specialized hospital. One-year follow-up}

\author{
Edgar Corpus-Mariscal' ${ }^{1}$, Arturo García-Galicia ${ }^{2 *}$, Daniel Esperanza-González ${ }^{1}$, Álvaro J. Montiel-Jarquin², \\ José J. Torreblanca-Reyes ${ }^{2}$ y Carlos F. Morales-Flores ${ }^{1}$
}

${ }^{1}$ Servicio de Columna, Unidad Médica de Alta Especialidad, Hospital de Traumatología y Ortopedia; ${ }^{2}$ División de Investigación, Unidad Médica de Alta Especialidad, Hospital de Especialidades de Puebla. Centro Médico Nacional “General de División Manuel Ávila Camacho", Instituto Mexicano del Seguro Social, Puebla, México

\section{Resumen}

Introducción: La mortalidad anual de la fractura de cadera va de del 15 al 20\%. El pronóstico independiente del tratamiento es la presencia de enfermedades sistémicas. La pronta solución quirúrgica disminuye la mortalidad. Los contraindicantes de cirugía elevan el riesgo de muerte. Objetivo: Describir la supervivencia a un año de pacientes mayores de 60 años con fractura de cadera manejados con tratamiento conservador vs. quirúrgico en el Hospital de Traumatología y Ortopedia. Métodos y materiales: Estudio comparativo, transversal, en mayores de 60 años con fractura de cadera. Se evaluó edad, sexo, diagnóstico, incidencia, prevalencia, pronóstico, comorbilidades y lado predominante. Se utilizó estadística descriptiva. Resultados: Cien pacientes, 29 hombres, 71 mujeres. Lado predominantemente afectado: izquierdo; fractura más frecuente: transtrocantérica; 71 pacientes recibieron tratamiento quirúrgico, el 75\% sobrevivieron al año posterior a la fractura. Veintinueve pacientes recibieron tratamiento conservador, el 55\% sobrevivieron al año posterior a la fractura. La fractura de cadera registró mayor mortalidad en pacientes con comorbilidades asociadas. La supervivencia al año posterior a la fractura es mayor en los pacientes con tratamiento quirúrgico. Conclusión: La mortalidad al año de los pacientes mayores de 60 años con fractura de cadera fue del $25 \%$. El tratamiento quirúrgico mostró mayor supervivencia.

Palabras clave: Supervivencia. Mortalidad. Fractura de cadera. Tratamiento.

\section{Abstract}

Background: Hip fracture mortality goes from 15-20\%. Prognosis independent of treatment is the presence of systemic diseases. The prompt surgical solution reduces mortality. Contraindicants for surgery raise the risk of death. Aim: Describe the one-year survival of patients older than 60 years with hip fracture managed with conservative versus surgical treatment in a third level Hospital. Material and Methods: Comparative, homodemic, cross-sectional study of those over 60 years with hip fracture. Age, gender, diagnosis, incidence, prevalence, prognosis, comorbidities and predominant side were evaluated. Descriptive data were used. Results: 100 patients were recruited, 29 men, 71 women. The predominantly affected side: left; the most frequent fracture: transtrochanteric; 71 patients received surgical treatment, $75 \%$ survived the year after the fracture. 29 patients received conservative treatment, 55\% survived the year after the fracture. Hip fracture resgistered higher mortality in patients with associated comorbidities. Survival one year after fracture is greater in patients with surgical treatment. Conclusion: One-year mortality of patients over 60 years with hip fracture was $25 \%$. Surgical treatment showed greater survival.

Key words: Hip fracture. Survival. Mortality. Treatment.

Correspondencia:

*Arturo García-Galicia

E-mail: neurogarciagalicia@yahoo.com.mx
Disponible en internet: 13-05-2021 Rev Hosp Jua Mex. 2021;88(3):100-104

www.revistahospitaljuarez.com 1405-9622/C 2020 Sociedad Médico-Quirúrgica del Hospital Juárez de México, A.C. Publicado por Permanyer. Este es un artículo open access bajo la licencia CC BY-NC-ND (http://creativecommons.org/licenses/by-nc-nd/4.0/). 


\section{Introducción}

La fractura de cadera en el adulto mayor es una eventualidad que resulta devastadora en la mayoría de los casos. Afecta al equilibrio físico, mental, funcional y social ${ }^{1}$. Se espera un incremento de esta patología, asociado al aumento de la población de adultos mayores $^{2}$.

Es la principal causa de hospitalización en los servicios de urgencias ortopédicos, con hasta el $90 \%$ de los casos en mayores de 50 años ${ }^{1}$. Los principales factores causales son caídas, osteoporosis, cambios osteomusculares asociados con la edad, medicación con ansiolíticos o psicotrópicos (por disminución del estado de alerta del paciente), pérdida de la agudeza visual o estados de demencia senili ${ }^{3-5}$. Se calcula que a los 60 años el 15\% de la población sufre al menos una caída anual, a los 65 años un $28 \%$ y a los 75 años un $35 \%$.

Es más frecuente en mujeres que en hombres 6,7 . Las fracturas más comunes son las de cuello femoral y las transtrocantéricas ${ }^{1}$.

Entre el 2002 y el 2007 en México se reportaron 71,771 egresos hospitalarios por causa de fractura de fémur, reportándose un $47.2 \%$ en individuos de 65 años o mayores $(69.5 \% \text { en mujeres) })^{8,9}$.

Por otra parte, se considera que una de cada cuatro personas adultas cursa con osteopenia u osteoporosis, por lo que se estima que entre 1 y 2 de cada 10 mujeres tendrán una fractura de cadera a lo largo de su vida².

Entre el 23.6 y el $34.5 \%$ de las personas con fractura de cadera fallecen después del primer año². Influyen factores como sexo, edad, comorbilidad asociada, estado funcional, deterioro mental, tipo de fractura, características del entorno social habitual y circunstancias como vivir solo o acompañado $0^{3,8-13}$.

En promedio la mortalidad por fractura de cadera va del 15 al $20 \%{ }^{14-16}$. Por otra parte, se estima que la mortalidad durante la fase hospitalaria aguda va del 2 al $7 \%$, mientras que del 6 al $12 \%$ durante el mes posterior y entre 17 y el $33 \%$ al cabo del primer año tras la fractura. En hombres oscila entre el 32 y el $62 \%$ al año de la fractura, en las mujeres se sitúa entre el 17 y el $29 \%{ }^{17}$.

Se ha demostrado que el mejor tratamiento es el quirúrgico, el cual disminuye la mortalidad, mientras que el riesgo se duplica por cada dos días de espera quirúrgica. El manejo conservador implica mayor estancia hospitalaria y menor retorno de los pacientes al nivel funcional previo. Sin embargo, en la mayoría de los casos la mortalidad y tardanza del tratamiento quirúrgico en realidad son producto de la comorbilidad ${ }^{13-19}$.
El objetivo de este trabajo fue analizar la supervivencia a un año de pacientes mayores de 60 años con fractura de cadera manejados con tratamiento conservador vs. quirúrgico en el Hospital de Traumatología y Ortopedia.

\section{Material y métodos}

El presente trabajo fue previamente aprobado por el Comité Local de Investigación y Ética en Investigación en Salud n. ${ }^{\circ} 2105$ (registro: R-2018-2105-004).

Estudio comparativo, retrospectivo, longitudinal, realizado en una unidad médica especializada en traumatología y ortopedia del Instituto Mexicano del Seguro Social en Puebla, en pacientes con fractura de cadera, durante mayo de 2017 a mayo de 2018. Se incluyeron pacientes de 60 años o más, de ambos sexos, con fractura de cadera tratados de forma quirúrgica y no quirúrgica. Se excluyeron pacientes con lesiones previas de cadera. Se eliminaron los pacientes con expediente clínico incompleto, muerte intrahospitalaria o con pérdida del seguimiento anual. Se evaluaron edad, sexo, diagnóstico, incidencia, prevalencia, pronóstico, comorbilidades, lado predominante y osteopenia.

Para el análisis de los datos se utilizó estadística descriptiva.

\section{Resultados}

Se incluyeron 100 pacientes. La mayor parte fueron mujeres. Las edades por sexo y tratamiento se muestran en la tabla 1.

La mayoría de los pacientes estudiados recibieron tratamiento quirúrgico. El lado izquierdo predominó como el lado más afectado. Por su parte, el tipo de fractura que más se presentó fue la transtrocantérica (Tabla 1).

De los 69 pacientes que sobrevivieron a un año posterior a la fractura de cadera, el 77\% (53 pacientes) habían recibido tratamiento quirúrgico y un $23 \%$ (16 pacientes) tratamiento conservador (Fig. 1).

Apenas más de la mitad de los pacientes que recibieron tratamiento conservador (16,55\%) sobrevivieron un año posterior a la fractura. De los 71 pacientes manejados con tratamiento quirúrgico, 53 (75\%) sobrevivieron a un año posterior a la fractura.

Del total de la población, la mayoría de los pacientes tenían al menos una comorbilidad asociada (esta relación se muestra en la tabla 1), el mismo patrón se mantiene en ambos abordajes terapéuticos. 
Tabla 1. Características de los pacientes con fractura de cadera en el Hospital de Traumatología y Ortopedia de Puebla

\begin{tabular}{|c|c|c|c|}
\hline \multicolumn{4}{|c|}{ Edad promedio por sexo (años)* } \\
\hline \multicolumn{2}{|c|}{$\begin{array}{l}\text { Hombres }(n=71) \\
81.4(62-95)( \pm 8.1)\end{array}$} & $\begin{array}{l}\text { Mujeres }(n=29) \\
99(59-95)( \pm 8.9)\end{array}$ & $\begin{array}{c}\text { Total }(n=100) \\
79.73(59-95)( \pm 8.9)\end{array}$ \\
\hline \multicolumn{4}{|c|}{ Tratamiento } \\
\hline \multicolumn{2}{|c|}{$\begin{array}{l}\text { Tratamiento conservador } \\
229(29 \%)\end{array}$} & \multicolumn{2}{|c|}{$\begin{array}{c}\text { Tratamiento quirúrgico } \\
71(71 \%)\end{array}$} \\
\hline \multicolumn{4}{|c|}{ Comorbilidades } \\
\hline $\begin{array}{c}\text { Sin } \\
\text { comorbilidades } \\
18(18 \%)\end{array}$ & $\begin{array}{c}\text { Una } \\
33(33 \%)\end{array}$ & $\begin{array}{l}\text { Dos } \\
25(25 \%)\end{array}$ & $\begin{array}{l}\text { Tres o más } \\
24(24 \%)\end{array}$ \\
\hline \multicolumn{4}{|c|}{ Tipo de fractura } \\
\hline \multicolumn{2}{|c|}{$\begin{array}{c}\text { Transtrocantérica } 67 \\
(67 \%)\end{array}$} & \multicolumn{2}{|c|}{$\begin{array}{c}\text { Transcervicales } 33 \\
(33 \%)\end{array}$} \\
\hline
\end{tabular}

*La edad está expresada en media (mínimo-máximo) (desviación estándar).

Tabla 2. Edad* y comorbilidades de según el tratamiento

\begin{tabular}{|c|c|}
\hline \multicolumn{2}{|c|}{ Edad promedio } \\
\hline Tratamiento conservador & Tratamiento quirúrgico \\
\hline 77.8 años $(62-95)( \pm 8.5)$ & 80.5 años $(60-94)( \pm 8.9)$ \\
\hline Tratamiento conservador \\
\hline Comorbilidades asociadas & Sin comorbilidades \\
\hline $82(82 \%)$ & $17(17 \%)$ \\
\hline Comorbilidades asociadas & Sin comorbilidades \\
\hline $81(81 \%)$ & $18(18 \%)$ \\
\hline
\end{tabular}

*La edad está expresada en media (mínimo-máximo) (desviación estándar).

En la figura 2 se ilustra la supervivencia y mortalidad a un año en hombres y mujeres; en ambos rubros predominaron las mujeres.

La relación de tratamiento quirúrgico vs. conservador se ven en la tabla 1, así como el porcentaje de pacientes con o sin comorbilidades y los tipos de fractura más frecuentes.

La mortalidad posterior a la fractura se muestra en la figura 3.

La edad promedio y el porcentaje de pacientes con comorbilidades asociadas para ambos tratamientos se muestran en la tabla 2.

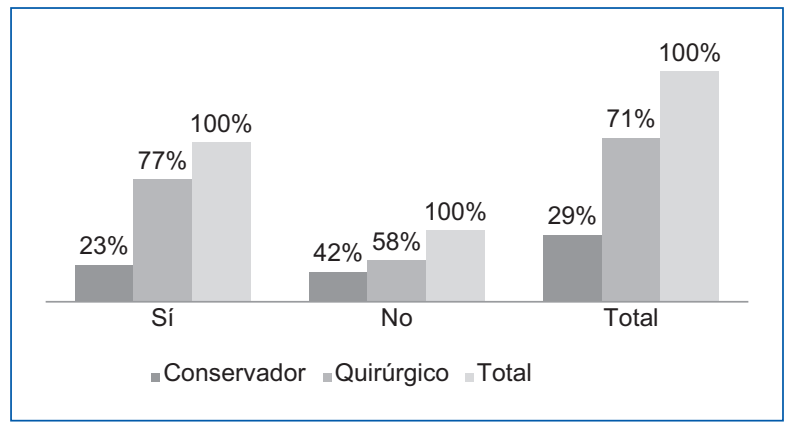

Figura 1. Supervivencia a un año de acuerdo con el tratamiento.

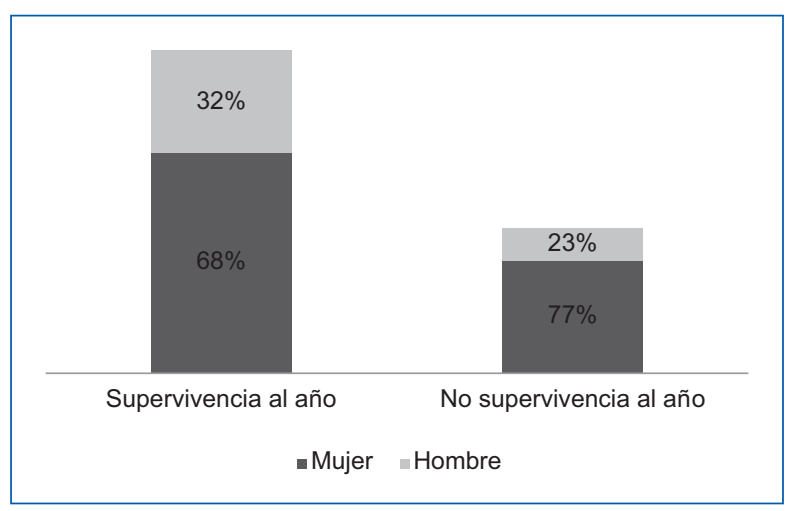

Figura 2. Supervivencia y mortalidad por sexo a un año. Los porcentajes para supervivencia al año equivalen a: $68 \%$, 47 pacientes, y $32 \%, 22$ pacientes. No supervivencia al año equivale a: $23 \%, 7$ pacientes, y $77 \%$, 24 pacientes.

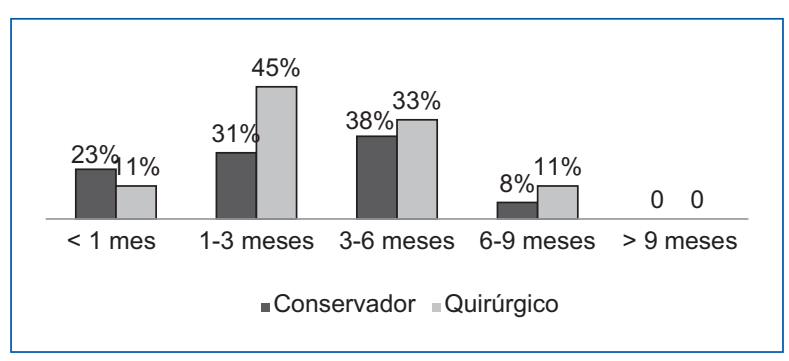

Figura 3. Mortalidad de acuerdo con el tratamiento por trimestres.

De los 29 pacientes que recibieron tratamiento conservador, seis tuvieron un riesgo quirúrgico Goldman II y ASA II (clasificación empleada por la American Society of Anesthesiologists), nueve pacientes tuvieron clasificación Goldman II y ASA III, y 14 pacientes tuvieron un riesgo quirúrgico Goldman III o IV (Tabla 3). 
Tabla 3. Riesgo quirúrgico y tratamiento de los pacientes con fractura de cadera en el Hospital de Traumatología y Ortopedia de Puebla

\begin{tabular}{|l|c|c|c|}
\hline Riesgo quirúrgico & Conservador & Quirúrgico & Total \\
\hline ASA II Goldman II & 6 & 52 & 58 \\
\hline ASA II Goldman III & 1 & 0 & 1 \\
\hline ASA III Goldman II & 9 & 17 & 26 \\
\hline ASA III Goldman III & 12 & 2 & 14 \\
\hline ASA IV Goldman III & 1 & 0 & 1 \\
\hline Total & 29 & 71 & 100 \\
\hline
\end{tabular}

ASA: (clasificación empleada por la) American Society of Anesthesiologists.

Del total de pacientes, 31 no sobrevivieron después de un año de la fractura, 18 recibieron tratamiento quirúrgico y 13 tratamiento conservador.

De los que se optó por la resolución quirúrgica, en su mayoría tenía clasificación ASA II y Goldman II. Mientras que más de la mitad de los que recibieron tratamiento conservador tenía clasificación de ASA III y Goldman III. La mayoría de los pacientes que murieron antes del año tenían por lo menos una comorbilidad asociada.

\section{Discusión}

La población de adultos mayores ha aumentado exponencialmente en las últimas décadas, con la asociación de enfermedades crónicas como la osteoporosis y sus consecuencias, incluyendo las fracturas de cadera $^{2-6}$. Así, la fractura de cadera es la causa más común de hospitalización en los servicios de urgencias ortopédicos. La incidencia incrementa exponencialmente con la edad, ocurriendo el $90 \%$ en mayores de 50 años $^{1-3}$. El promedio reportado en la bibliografía internacional es de 80 años $^{3}$, similar a los resultados del presente trabajo (79.3 años).

En cuanto al sexo, este estudio coincide con otros reportes con el sexo femenino mayormente afectado $^{2,5,7}$. Se ha comentado que en las mujeres la pelvis ósea es más ancha y con tendencia a la coxa vara, además de que después de los 60 años tienden a disminuir su actividad, adquieren osteoporosis más jóvenes y su promedio de vida es mayor que la de los hombres. Todos estos factores ocasionan el incremento en la frecuencia sea mayor en mujeres que en hombres?.
El tipo de fractura más frecuente reportada es la transtrocantérica, con una incidencia del $56.6 \%^{12,13}$, al igual que en este trabajo, si bien con un porcentaje mayor (67\%).

En cuanto a la mortalidad al año después de la fractura nuestro estudio arrojó cifras mayores (31\%) a lo mencionado por la literatura consultada $(15-20 \%)^{11}$. La mayoría de los pacientes de nuestro estudio tenía por lo menos una comorbilidad asociada, mientras que la minoría se reporta aparentemente sana o sin comorbilidades asociadas (18\%). La principal enfermedad asociada fue hipertensión arterial (61\%), seguida de diabetes mellitus tipo 2 (42\%).

Las alteraciones fisiológicas y del metabolismo asociadas a la fractura de cadera generan condiciones que contraindican el tratamiento quirúrgico, siendo las comorbilidades el principal factor de demora para llevar a cabo el tratamiento quirúrgico ${ }^{13-18}$. En pacientes con enfermedad cardiovascular, el riesgo aumenta entre 2.5 y 4 veces en la cirugía de urgencia en comparación con la cirugía programada. Sin embargo, la cirugía de urgencia parece guardar una relación importante con las complicaciones en el postoperatorio, aun después de haber controlado la enfermedad subyacente ${ }^{17}$. En el presente estudio, el riesgo quirúrgico elevado, Goldman III (riesgo de complicaciones mayores 11\%, muerte cardiaca 0-2\%) o IV (riesgo de complicaciones mayores $22 \%$, muerte cardiaca $56 \%$ ) se asoció a optar por el tratamiento no quirúrgico.

Mención aparte merecen los pacientes que eran candidatos a tratamiento quirúrgico y que tenían bajo riesgo quirúrgico, pero prefirieron el tratamiento conservador (15\%).

Las enfermedades concomitantes frecuentemente son en sí mismas contraindicaciones para el tratamiento quirúrgico y, por lo tanto, el tratamiento conservador es obligado ${ }^{18}$. Los resultados que hoy se presentan indican mayor supervivencia a un año después de la fractura en pacientes con manejo quirúrgico, en comparación con los reportes internacionales ${ }^{18-21}$.

El tratamiento conservador se relaciona con mayor mortalidad en pacientes mayores de 60 años $^{19}$. En la presente serie, la mayor mortalidad en pacientes no quirúrgicos se registró entre los meses 1 y 3 después de la fractura. Este manejo implica mayor estancia hospitalaria y complicaciones propias de las comorbilidades.

Existen diferentes indicadores (densidad mineral ósea, puntaje T) e instrumentos (FRAX) que ayudarían a implementar medidas preventivas en una población cada vez más numerosa ${ }^{20-24}$. 
Los resultados del presente trabajo sugieren que la elección del abordaje quirúrgico en los pacientes con fractura de cadera se asocia con mayor supervivencia a un año. Las comorbilidades frecuentemente determinan la contraindicación.

En pacientes con manejo conservador, los primeros tres meses son de mayor riesgo de mortalidad.

\section{Financiamiento}

La presente investigación no ha recibido ninguna beca específica de agencias de los sectores público, comercial o sin ánimo de lucro.

\section{Conflicto de intereses}

Los autores declaran que no existe conflicto de intereses.

\section{Responsabilidades éticas}

Protección de personas y animales. Los autores declaran que para esta investigación no se han realizado experimentos en seres humanos ni en animales.

Confidencialidad de los datos. Los autores declaran que han seguido los protocolos de su centro de trabajo sobre la publicación de datos de pacientes.

Derecho a la privacidad y consentimiento informado. Los autores han obtenido el consentimiento informado de los pacientes $y / 0$ sujetos referidos en el artículo.

\section{Bibliografía}

1. Negrete Corona J, Alvarado Soriano JC, Reyes Santiago LA, Fractura de cadera como factor de riesgo en la mortalidad en pacientes mayores de 65 años. Estudio de casos y controles. Acta Ortop Mex 2014; 28(6): 352-362.
2. Viveros-García JC, Torres-Gutiérrez JL, Alarcón-Alarcón T, et al. Fractura por fragilidad en México ¿En dónde estamos hoy? ¿Hacia dónde queremos ir? Acta Ortop Mex 2018 32(6) 334-341.

3. Valles Figueroa JFG, Malacara Becerra M, Gómez Mont Landerache G, et al. Tratamiento quirúrgico de las fracturas de cadera. Acta Ortop Mex 2010; 24(4):242-247.

4. Van de Ree CLP, Landers MJF, Kruithof N, De Munter L, et al. Effect of frailty on quality of life after hip fracture: a longitudinal study. BMJ Open 2019;9 1-8

5. Rego-Hernández JJ, Hernández-Seuret CA, Andreu-Fernández MA, et al. Factores asociados a la fractura de cadera en el hospital clínicoquirúrgico "Dr. Salvador Allende". Rev Cubana Salud Pública. 2017;43(2);149-165.

6. Muñoz S, Lavanderos J, Vilches L, et al. Fractura de cadera. Cuad Cir. 2008;22(19):73-81.

7. Collazo Álvarez H, Boada Sala NM. Morbimortalidad por fractura de cadera. Rev. Cubana Ortop. Traumatol. 2000;14(1-2): 21-5.

8. Brunner LC, Eshilian Oates L, Kou TY. Kuo TY. Hip fractures in adults. Am Fam Physician. 2003;67(3):537-42.

9. Etxebarria-Foronda Í. Incidencia de la fractura de cadera en España. Med Clin (Barc) 2015;145(11):482-4

10. Vestergaard $P$, Rejnmark $L$, Mosekilde L. Loss of life years after a hip fracture. Acta Orthop 2009;80(5):525-530.

11. Bergström U, Jonsson $H$, Gustafson $Y$, et al. The hip fracture incidence curve is shifting to the right. A forecast of the age-quake. Acta et al 2009; 80(5):520-4

12. Handoll HH, Parker MJ. Conservative versus operative treatment for hip fractures in adults. Cochrane Database Syst Rev. 2008 Jul 16:(3):CD000337.

13. Meinberg EG, Agel J, Roberts CS, et al. Fracture and Dislocation Classification Compendium-2018. J Orthop Trauma. 2018 Jan;32 Suppl 1:S1-S170.

14. Vestergaard P, Rejnmark L, Mosekilde L. Loss of life years after a hip fracture. Acta Orthop. 2009; 80(5):525-530.

15. Dodd AC, Bulka C, Jahangir A, et al. Predictors of 30-day mortality following hip/pelvis fractures. Orthop Traumatol Surg Res. 2016;102(6):707-10.

16. Gandhi R, Perruccio AV. Reducing mortality and morbidity following hip fracture: Is expedited surgery the way to go?. CMAJ. 2016;188(12):E277-E278.

17. Fernández Ramos $\mathrm{H}$, Pantoja Muñoz $\mathrm{HJ}$, Pozo Romero JA, et al. (2011). Valor predictivo del índice multifactorial de riesgo cardiaco de Goldman para cirugía no cardiaca. Rev Cuba Anestesiol Reanim 2011;10(2):91-100.

18. Palomino L, Ramírez R, Vejarano J, et al. Fractura de cadera en el adulto mayor: la epidemia ignorada en el Perú. Acta Med Peru. 2016;33(1):15-20.

19. Lu Y, Uppal HS. Hip fractures: Relevant Anatomy, Clasiffication, and Biomechanics of Fracture and Fixation. Geriatr Orthop Surg Rehabil. 2019;10:2151459319859139.

20. Berry SD, Kiel DP, Colón-Emeric C. Hip fractures in older adults in 2019. JAMA. 2019;321(22):2231-2232.

21. Pollmann CT, Røtterud JH, Gjertsen JE, et al. Fast track hip fracture care and mortality - an observation an observational study of 2230 patients. BMC Musculoskelet Disord. 2019;20(1):248.

22. Bhandari M, Swiontkowski M. Management of Acute Hip Fracture. N Engl J Med 2017;377:2053-2062.

23. Okike K, Chan PH, Prentice HA, et al. Association between uncemented vs cemented hemiarthroplasty and revision surgery among patients with hip fracture. JAMA. 2020;323(11):1077-1084.

24. Gjertsen JE. Should Total Hip Arthroplasty Be Used for Hip Fracture?. N Engl J Med. 2019;381(23):2261-2262. 